Pathologe 2021 - 42 (Suppl 2):S149-S154 https://doi.org/10.1007/s00292-021-00996-9 Angenommen: 3. September 2021 Online publiziert: 8. Oktober 2021 ๑) Springer Medizin Verlag GmbH, ein Teil von Springer Nature 2021

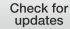

\section{Modellsysteme in der gastroenterologischen Forschung}

\author{
Vom Tiermodell über humane Organoide in die Klinik \\ Frank Arnold · Alexander Kleger \\ Abteilung für Innere Medizin I, Universitätsklinikum Ulm, Ulm, Deutschland
}

\title{
Zusammenfassung
}

Über die letzten Jahrzehnte haben sich verschiedene Modelle in der gastroenterologischen Forschung etabliert, die erheblich dazu beigetragen haben die (patho-)physiologischen Prozesse verschiedener gastrointestinaler (GI) Krankheiten (Entzündungen, Organverletzungen, Karzinome) besser zu verstehen. Dieser Review konzentriert sich auf solche unterschiedlichen Modelle, darunter genetisch veränderte Mausmodelle (GVMM), Xenografts und organoidbasierte Kultursysteme. GVMMs legten dabei den Grundstein zur erfolgreichen Modellierung dieser Krankheiten. Diese haben den entscheidenden Vorteil, dass Erkrankungen in ihrer physiologischen Umgebung beurteilt werden können und erlauben so den Einfluss verschiedener Zelltypen (Epithel, Fibroblast, Immunzellen) zu untersuchen. Die Diskrepanz zwischen Maus und Mensch beinhaltet jedoch einen entscheidenden Nachteil, der zumindest teilweise durch Transplantation humaner Zellen in immungeschwächten Wirtstieren umgangen werden konnte. Die zeit- und arbeitsintensive Generierung von solchen Xenograftmodellen schränkt jedoch deren Nützlichkeit für ein zeitnahes präklinisches Screening erheblich ein. Neuartige organoidbasierte humane Zellkultursysteme aus adulten Stammzellen oder pluripotenten Stammzellen sind ein vielversprechendes humanes Tool zur Modellierung von Gl-Erkrankungen. Bereits heute zeigen erste Ergebnisse deren Nützlichkeit in der Regulation adulter Gewebehomöostase, Regeneration und Tumorentwicklung. Darüber hinaus lässt sich dieses System einfach in der klinischen Diagnostik für Tumorpatienten etablieren und ermöglicht so eine zeitnahe Ex-vivo-Pharmakotypisierung, um personalisierte Therapiestrategien zu entwickeln.

\section{Schlüsselwörter}

Mausmodelle $\cdot$ Xenografts · Organoide $\cdot$ Personalisierte Medizin · Organ-on-a-Chip

Über die letzten Jahre haben sich verschiedene Modelle in der Forschung etabliert, die erheblich dazu beigetragen haben, die Pathogenese diverser gastrointestinaler (GI) Krankheiten (z.B. Entzündungen, Organverletzungen, Karzinome) besser zu verstehen. Diverse Mausmodelle legten dabei den Grundstein zur erfolgreichen Modellierung dieser Krankheiten. Neuartige organoidbasierte humane Zellkultursysteme sind jedoch bereits heute ein vielversprechendes Tool in der personalisierten Medizin und könnten zukunftsorientiert die Modellierung von GI-Erkrankungen in einem humanen System revolutionieren.
Gastrointestinale Krankheiten können in vielen Fällen immer noch einen unerwarteten Krankheitsverlauf haben und gehen in vielen Fällen (z. B. Karzinome) mit einer schlechten Prognose einher. Gl-Krankheiten (z.B. Infektionen, Entzündungen, Organverletzungen, Karzinome), die mit einem heterogenen Krankheitsbild verbunden sind, zeichnen sich durch eine komplexe Pathophysiologie (z. B. Dysplasie, Fibrose) aus, die durch verschiedene genetische und molekulare Veränderungen stark beeinflusst werden. Darüber hinaus sind diese Erkrankungen geprägt von einem temporären bzw. stadiumabhängigen Zusammenspiel zwischen unterschiedlichen Zelltypen (z. B. Epithel, Fibroblasten, Im- 
munzellen). Diese Faktoren wirken sich erheblich auf die Behandlung der Krankheit und damit den Ausgang aus.

Die gastrointestinale Forschung hat von der Verfügbarkeit verschiedener Mausmodelle (genetisch veränderte Mausmodelle [GVMMs], Xenografts) und neuerdings exvivo-organoid-basierten Kultursystemen in den letzten Jahren enorm profitiert (- Abb. 1). Die Einbindung solcher verschiedener Modelsysteme und der technologische Fortschritt (z.B. Transkriptom, Proteom) ermöglichten es, die räumlichen und zeitlichen Veränderungen (z.B. Dysplasie und Fibrose) während der Entwicklung und des Verlaufs verschiedener Gl-Erkrankungen in einem genotypspezifischen Kontext zu analysieren. Darüber hinaus können diese Tools als zuverlässige Vorhersagemodelle genutzt werden, um potenziell wirksame Medikamente mit tolerierbarer Toxizität für bestimmte GIErkrankungen wie Tumorerkrankungen zu finden und so die schlechte Prognose von Patienten zu verbessern.

\section{Genetisch veränderte Maus- modelle in der GI-Forschung}

GVMMs ermöglichen es den Einfluss individueller Gene zum einen auf die embryonale Entwicklung $[25,35]$ und zum anderen auf Krankheitsverläufe (z.B. Entzündungen, Organverletzungen, Tumorentwicklung) zu untersuchen. Verschiedene Mausmodelle [7], nämlich (i) konstitutive oder konventionelle Knockout-Mäuse, (ii) gewebespezifische und/oder zeitlich abhängige konditionale Knockout- und KnockinMäuse haben sich hierbei in den letzten Jahrzehnten in der Gl-Forschung etabliert (-Abb. 1). So konnte zum Beispiel durch die Verwendung eines konstitutiven Knockouts in Kombination mit experimentell induziertem Leberversagen (Tetrachlormethan) oder einer Pankreatitis (Caerulein) gezeigt werden, dass Dickkopf 3, ein Regulator des Wnt-Signalwegs, die Regeneration stark einschränkt und somit potenziell als neuartige Therapie bei GlOrganverletzungen genutzt werden könnte [2].

Die Entwicklung von Gl-Karzinomen (z. B. kolorektales Karzinom, Pankreaskarzinom) ist unter anderem durch Mutationen in verschiedenen Onkogenen und Tumor- suppressorgenen verursacht. Knockinund Knockout-Mäuse ermöglichen einen gewebespezifischen Einbau onkogener Treibermutationen bzw. das Entfernen von Tumorsuppressorgenen, wodurch gezielt die Entwicklung bestimmter GlKarzinome mit ähnlicher Pathogenese wie im Menschen erlangt werden kann. Zum Beispiel widerspiegeln GVMMs des kolorektalen Karzinoms die Pathogenese von sporadischem sowie vererbten kolorektalem Karzinom. Jedoch hat sich hier herausgestellt, dass es bei diesen Mausmodellen, im Gegensatz zum humanen Tumor, an Invasivität, Metastasierung und Tumorheterogenität fehlte. Dennoch zeigten die GVMMs des kolorektalen Karzinoms einen Nutzen für die Untersuchung der Tumormikroumgebung sowie für systemische Immunantworten und unterstützten so die Entwicklung neuer therapeutischer Ansätze [5].

Die pankreasspezifische (PTF1a-Cre, PDX1-Cre) LSL-Kras ${ }^{\text {G12D-Knock-in-Maus }}$ (KC-Modell) dahingegen war die erste genetisch veränderte Maus, die die erwartete Progression von pankreatischen intraepithelialen Neoplasien (PanIN) zu metastatischen Tumoren im Pankreas zeigte. Hierbei war die Tumorentwicklung mit den gleichen Signalwegen verbunden, die auch bei einem humanen Pankreaskarzinom beobachtet worden waren [13]. In einer erst kürzlich veröffentlichten Studie konnte mit dem KC-Modell gezeigt werden, dass die Grundlage der Tumorentwicklung durch die Dosierung von mutiertem Kras innerhalb von Vorläuferläsionen (PanINs) gegeben ist, was sowohl den Grad der Karzinogenese als auch die Metastasierung im späteren Stadium definiert [22]. Das KC-Modell zeigt jedoch eine relative lange Latenzzeit und nur einen relativ geringen Grad an Metastasierung auf, wodurch es sich zwar ideal eignet, um die einzelnen Stadien der Tumorentwicklung zu untersuchen, aber nicht vollständig dem humanen PDAC gleichkommt. Die zusätzliche Inaktivierung des Tumorsuppressorgens Trp53 (Trp ${ }^{R 172 H}$-Mutation, KPC-Modell) [7, 14] führte hierbei zu einem Phänotyp mit beschleunigtem Tumorfortschreiten, vermehrter Metastasierung und deutlich verkürztem Gesamtüberleben; und kommt daher der Tumorbiologie eines humanen PDAC deutlich näher. Darüber hinaus zeigt das KPC-Mausmodell eine ähnliche Tumormikroumgebung wie das humane PDAC einschließlich verschiedener tumorassoziierte Fibroblasten (myCAF, iCAF, apCAF) [8] und dem Immunzellprofil [30] und eignet sich daher ideal, um den Einfluss und das Zusammenspiel zwischen Tumor und Stroma zu untersuchen. Durch die Verwendung von zelltypspezifischen (azinar: Ptf1a bzw. duktal: Sox9) zeitlichinduzierbaren Cre-Rekombinasen mit Tamoxifen konnte zudem der Einfluss der kontrovers diskutierten Ursprungszelle auf die Entwicklung des PDACs im KPC-Modell untersucht werden. Lee und Kollegen zeigten hierbei, dass die Tumorentwicklung deutlich schneller und mit hochgradigen PanIN-Läsionen in Mäusen mit duktalem Ursprung verbunden war. Im Gegensatz dazu wiesen PDACs mit azinärem Ursprung eine normale Dysplasie mit sowohl niedrig- als auch hochgradigen PanINs mit verzögerter Progression zu PDAC auf [21]. Da das KPC-Modell ein eigenständiges, immunkompetentes Modell darstellt, konnte es zudem erfolgreich zur präklinischen Bewertung von Inhibitoren, Immuntherapien und zur Verbesserung verschiedener Chemotherapien verwendet werden [34].

Neben den bereits erwähnten KCund KPC-Modellen haben sich mehr als 30 verschiedene GVMMs etabliert, die die am häufigsten gefundenen Mutationen in Pankreaskarzinom abdecken (z.B. SMAD4, CDKN2A) [34]. Diese dienten zur Untersuchung der Tumorentwicklung, Metastasierung sowie die Beteiligung der Tumormikroumgebung an der Karzinogenese [34] und reflektieren zudem Eigenschaften diverser PDAC-Gruppen, wie z. B. dem humanen instabilen PDAC, das durch Mutationen in DNA-Reparaturgenen (z. B. ATM) charakterisiert ist [24, 26].

\section{Xenografts als Modell zur Vorher- sage des Therapieansprechens bei Karzinomen}

Obwohl GVMMs besonders vorteilhaft sind, um die Rolle einzelner Mutationen im Krankheitsverlauf von Gl-Tumoren zu verstehen, stellt die Notwendigkeit, alle Zellen eines Zelltyps auf die gleiche Weise zu modifizieren, eine entscheidende Ein- 


\title{
Genetisch veränderte Mausmodelle
}

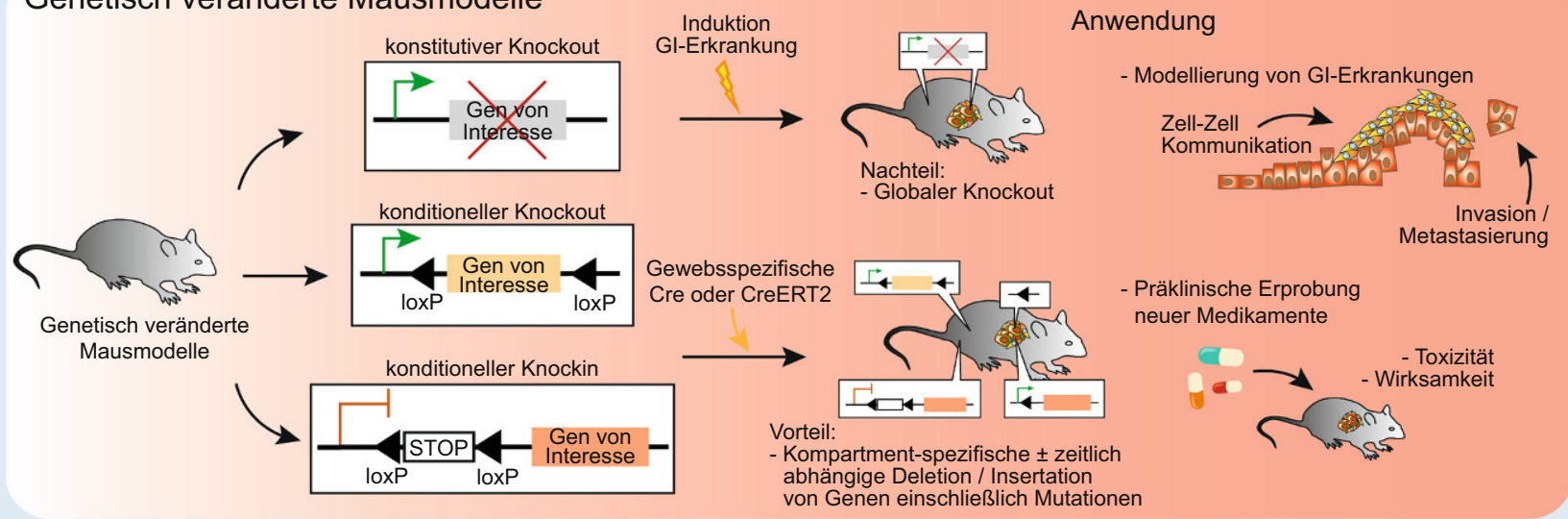

\section{Xenograft}

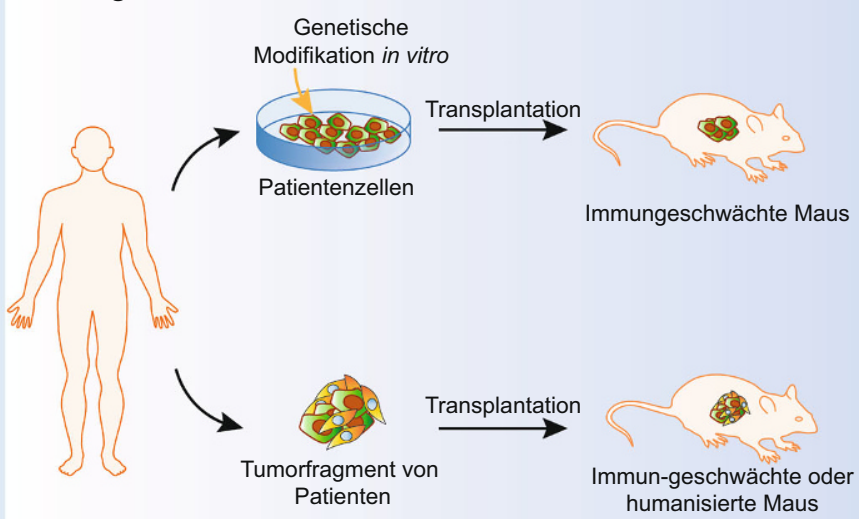

\section{Anwendung}

\begin{abstract}
- Einfluss einzelner Proteine oder
Medikamente auf Tumorwachstum
\end{abstract}

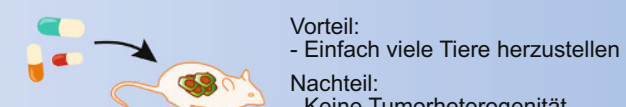

- Keine Tumorheterogenität

- Präklinische Modell

- Wirksamkeit neuartiger Therapieansätze auf Tumorwachstum - Vorhersage Therapieansprechen

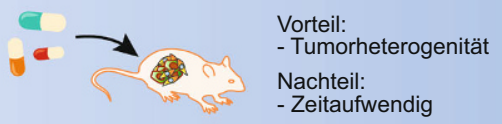

\section{Organoide}

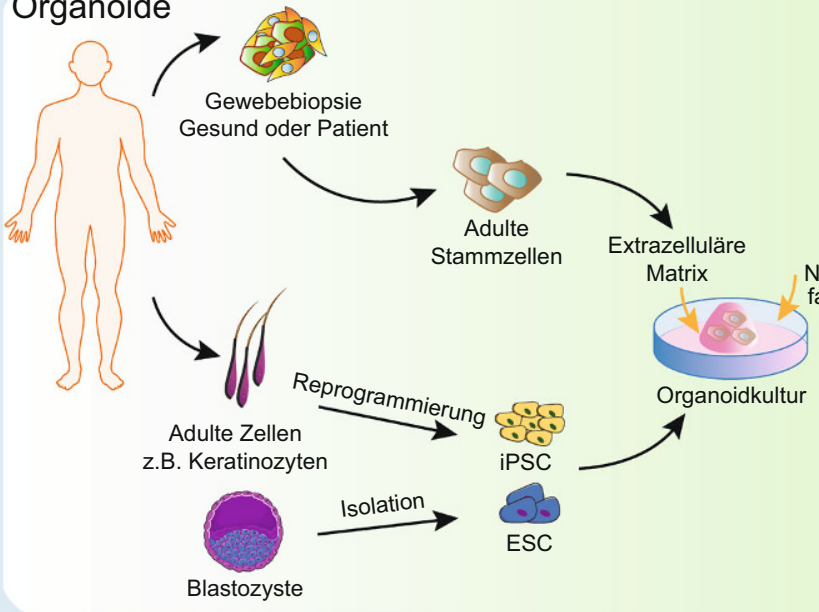

Anwendung

- Modellierung von GI-Erkrankungen

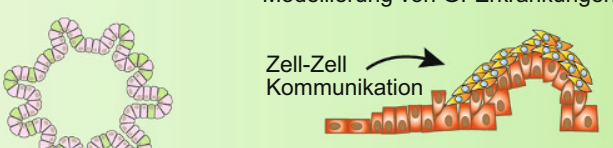

- High-Throughput Medikamenten-Screening
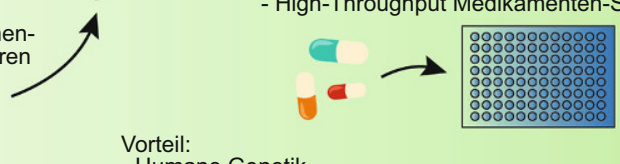

- Humane Genetik

- Einfache genetische Manipulation möglich (Gentherapie)

- Schnelle Translation in die Klinik (Therapieverbesserung)

Nachteil:

- Komplexe Ko-Kulturen (Epithel, Fibroblast, Immunzellen)

bzw. Organ-on-a-Chip erst teilweise realisierbar

- Komplexe und teure Protokolle für Organoide aus PSCs
.

Abb. 1 A Übersicht über verschiedene Modelle in der gastroenterologischen Forschung. Abbildung zeigt genetisch veränderte Mausmodelle, Xenografts und organoidbasierte Zellkultursysteme. ESC embryonale Stammzelle, GI gastrointestinal, iPSC induzierte pluripotente Stammzelle. Basierend auf Informationen aus Beutel et al. [3], Doyle et al. [7] und Weidenhofer et al. [34] wurde die Abbildung selbst generiert. Templates (z. B. PSCs) wurden teilweise von smart.servier.com lizenziert unter Creative-Common-Attribution-Lizenz (CC-BY3.0, https://creativecommons.org/licences/by/3.0/) entnommen 
schränkung dar, da sie nicht die normale Heterogenität eines humanen Tumors widerspiegelt. Der Verlust der intratumoralen Heterogenität in GVMMs kann mit dem Xenograftmodell umgangen werden. Hierbei werden entweder Patientenzelllinien oder ganze Tumorfragmente von Patienten (PDX) in immungeschwächte Mäuse implantiert (• Abb. 1). Die Verwendung von Patienten stammenden Zelllinien hat einen klaren Vorteil in der Fähigkeit, hunderte von Tieren aus derselben Zelllinie zu erzeugen. Dieses Modell eignet sich ideal, um den Einfluss einzelner Proteine auf das Tumorwachstum in seiner physiologischen Umgebung zu untersuchen [1]. Jedoch fehlt hierbei weiterhin die Heterogenität eines Patiententumors sowie die Wechselwirkungen mit den Zellen der Tumormikroumgebung des Ursprungsgewebes. Die Transplantation von ganzen Tumorfragmenten stellt ein besseres repräsentativeres Modell bereit, wodurch die eben erwähnten Nachteile teilweise überwunden werden können. Dementsprechend hat sich dies als leistungsfähiges Werkzeug in der Grundlagenforschung und translationalen Forschung erwiesen. PDX-Modelle bewahren biologische Merkmale des ursprünglichen Tumors sowie das komplexe Zusammenspiel mit der Tumormikroumgebung [9]. Dadurch kann das PDX-Modell gezielt als präklinisches Model genutzt werden. Zum einen ermöglichte dies, das Therapieansprechen neuartiger Behandlungsregime zu untersuchen [10]. Zum anderen konnte gezeigt werden, dass das Therapieansprechen in PDX-Modellen mit PDX-Tumor-erzeugten Organoiden [9] und Patienten mit gastrointestinalen Malignomen korrelierte [18].

Neuartigere PDX-Systeme verwenden außerdem humanisierte Wirtstiere mit menschlichem Immunsystem. Solch humanisierte Mäuse ermöglichen das komplexe Zusammenspiel verschiedener humaner Zelltypen, einschließlich immunologischer Aspekte, unterschiedlicher GI-Erkrankungen (z. B. Infektionen, Krebs) in einer physiologischen Umgebung zu analysieren und negieren die genetischen Unterschiede zwischen Menschen und GVMMs. Hierbei werden humane Zellen entweder aus peripherem Blut oder tumorinfiltrierenden Lymphozyten in im- mundefiziente Mäuse transplantiert. Dies geht allerdings mit einer erheblichen Graftversus-Host-Abstoßung einher. Alternativ können humanisierte Mausmodelle durch Transplantation von CD34+ humanen hämatopoetischen Stammzellen (HSC) oder Vorläuferzellen aus Nabelschnurblut generiert werden [33]. Die zeit- und arbeitsintensive Generierung von PDX-Mausmodellen schränkt jedoch deren Nützlichkeit gerade für ein zeitnahes präklinisches Screening mehrerer Medikamente entscheidend ein.

\section{Organoide aus adultem Gewebe als zentraler Meilenstein in der GI- Forschung}

Organoide sind in vitro miniaturisierte und vereinfachte Modellsysteme von Organen. Die Organoide stammen entweder aus geweberesidenten adulten Stammzellen (ASC) oder Tumorproben, die direkt aus Biopsieproben von Patienten gewonnen werden können. Solche Organoide gleichen stark dem In-vivo-Gewebe, was sie zu einem vielversprechenden Modellsystem für die Gewebehomöostase, Krankheitsmodellierung (z. B. Infektionen, Regeneration, Karzinome) und das Wirkstoffscreening macht (• Abb. 1).

In 2009 konnte das Labor von Hans Clevers die ersten komplexen 3D-organisierten Strukturen von ASC aus intestinalem Gewebe herstellen, indem die Zellen in einer extrazellulären Matrix mit gewebeähnlicher Steifheit und essenziellem Iöslichem Nischenfaktor kultiviert worden waren [27]. Intestinale Organoide werden heutzutage zunehmend wegen ihrer Fähigkeit geschätzt, wichtige architektonische und physiologische Merkmale der nativen Darmschleimhaut nachahmen zu können. Aus diesem Grund sind intestinale Organoide das vielseitigste und natürlichste In-vitro-Modell des Darms, um sowohl biologische Prozesse, die für die Aufrechterhaltung der Gewebehomöostase wichtig sind, als auch die Immunantwort nach enteropathogenem Befall zu untersuchen [11].

Mittlerweile können aus einer Vielzahl an verschiedenen Organen wie Leber, Magen oder Pankreas Organoide hergestellt werden. Die Isolation von Organoiden aus Patienten mit GI-Erkrankungen (z.B. he- reditären Erkrankungen, Karzinome) zeigte das enorme Potenzial dieses Tools im Bereich der personalisierten Medizin [23]. Die zystische Fibrose (ZF), eine hereditäre Erkrankung, die mit einer zähen Mukusbildung einhergeht, verursacht unter anderem Atemwegs- und Verdauungsprobleme und führt dadurch zu enormen Lebenseinschränkungen. Die Vielzahl an Genmutationen im verantwortlichen CFTR-Gen schränkt Behandlungserfolge zudem ein. Jedoch konnte mithilfe der Organoidtechnologie bei ZF-Patienten mit einem nicht charakterisierten seltenen CFTR-Genotyp ein Therapieansprechen auf die Behandlung mit Ivacaftor, einem CFTR-Potentiator, vorhergesagt werden [6]. Bei $97 \%$ aller ZF-Patienten lassen sich allerdings nur die Symptomatik und nicht die Ursache behandeln, da hier nur eine Gentherapie helfen würde. Interessanterweise konnte der Gendefekt in intestinalen Organoiden von 2 Patienten mit ZF durch Genmodifikation mit dem CRISPR/Cas9-System zumindest in vitro korrigiert werden [28], was einen möglichen Durchbruch für die Behandlung von ZF erbringen könnte.

Darüber hinaus spiegeln Organoide von Tumorpatienten die Heterogenität und Pathologie des primären Tumors wider $[3,9]$. Genom- sowie Transkriptomanalysen von patientenabstammenden Organoiden ermöglichten die Charakterisierung molekularer und funktioneller Subtypen von Bauchspeicheldrüsenkrebs, die letztendlich ein Ansprechen auf eine individualisierte Chemotherapie vorhersagen ließ [31]. Zudem konnte gezeigt werden, dass das In-vitro-Ansprechen von Organoiden auf Chemotherapeutika mit der klinischen Antwort bei Patienten mit Pankreaskarzinom, kolorektalem und gastroösophagealem Karzinom korrelierte [32]. Darüber hinaus haben sich Organoide als eine geeignete Methode zur Ex-vivo-Pharmakotypisierung in einem vernünftigen Zeitrahmen herausgestellt $[3,29]$ und sind daher PDX-Modellen deutlich überlegen.

\section{Differenzierte Organoide aus pluripotenten Stammzellen zur Modellierung von GI-Krankheiten}

Neben adulten Stammzellen können Organoide auch aus pluripotenten Stammzellen (PSCs) wie embryonalen Stammzellen 
(ESCs) oder induzierten PSCs (iPSCs) gewonnen werden (• Abb. 1). Induzierte PSCs werden dabei durch die Reprogrammierung differenzierter Zelltypen (z.B. mit OCT3/4, KLF4, SOX2, c-MYC) erhalten [19]. Initial waren Organoide, die aus menschlichen pluripotenten Stammzellen entwickelt wurden, besonders nützlich für die Herstellung von Geweben, bei denen die Zugänglichkeit zu adultem Gewebe erschwert ist, wie zum Beispiel Gehirn oder der Netzhaut [15]. Heutzutage profitiert auch die gastrointestinale Forschung von PSC-differenzierten organoidbasierten Kultursystemen. Gerade in der aktuellen COVID-19-Pandemie konnten humane intestinale Organoide aus PSCs wichtige Erkenntnisse über das Infektionsgeschehen aufdecken [12] und zeigten, dass SARSCoV-2 nur enteroendokrine Zellen und Paneth-Zellen, aber nicht Becherzellen infiziert [20]. Im Bereich von Pankreaserkrankungen konnten funktionelle azinäre und duktale pankreasähnliche Organoide (PDLOs) entwickelt werden [4, 16, 17]. Diese konnten erfolgreich zur Identifizierung neuer Biomarker für frühe Tumorstadien des Pankreaskarzinoms genutzt werden [36]. Darüber hinaus können durch Genveränderungen (z. B. Lentiviral, piggyBAC oder CRISPR/Cas9) hochindividuelle, patientenspezifische humane Organoidmodelle aus PSCs hergestellt werden [4, 17], die das Modellieren von GI-Krankheiten ermöglicht, was bis dahin nur in GVMMs realisiert werden konnte. $[4,36]$. So konnte beispielsweise gezeigt werden, dass die Expression des onkogenen KRAS in azinären Organoiden eine azinäre-zu-duktale metaplasieähnliche Veränderung in vitro induzierte [17]. PDLOs mit mutiertem $K R A S$ dahingegen zeigten ein reduziertes Wachstum einhergehend mit erhöhter epithelialer-zu-mesenchymaler Transition in vitro, was nach Transplantation in Mäuse zu differenzierten PDACs führte $[4,17]$. Im Gegensatz dazu führen transplantierte PDLOs mit mutiertem GNAS zu intraduktalen, papillären, muzinösen, neoplasienähnlichen Strukturen [4]. Diese Ergebnisse reflektierten die bereits bekannten Erkenntnisse aus Mausmodellen und beweisen, dass humane Organoidsysteme als alternative Systeme auch zur Modellierung von Gl-Erkrankungen genutzt werden können.
Ein noch immer limitierender Faktor dieser Organoide ist jedoch der fehlende Einfluss zellulärer Interaktionen mit unterschiedlichen Zelltypen (z. B. Epithel, Fibroblasten, Immunzellen). Diese zelluläre Kommunikation ist ein entscheidender Bestandteil der Pathogenese von Gl-Erkrankungen. Der Bereich „Organs-on-a-Chip" hat den Grundstein für die Entwicklung von solchen komplexeren Systemen in Kombination mit organoidbasierter Technologie gelegt. Synergistische Ansätze, die Organon-a-Chip-Setups und Organoide integrieren, zeigen (patho-)physiologische Ähnlichkeiten mit In-vivo-Systemen [15]. Beispielsweise konnte in in Mikrochips kultivierte PDLOs die metabolische Aktivierung von pankreatischen Sternzellen durch autokrine und parakrine Signale, welche infolge einer Inflammation, Verletzung oder Tumorentwicklung wesentlich zur Pathogenese von Pankreaserkrankungen beitragen, untersucht werden [36].

Obwohl es noch einige Hürden zu überwinden gibt, gerade in Bezug auf komplexere Kokultursysteme bestehend aus z. B. Organoiden, Fibroblasten und Immunzellen, hat die Organoidtechnologie bereits jetzt den Grundstein für die Modellierung von humanen Gl-Krankheiten gelegt und entscheidend die personalisierte Medizin vorangetrieben, wovon bereits heute der Patient profitiert.

\section{Fazit für die Praxis}

- Genetisch veränderte Mausmodelle (GVMMs) reflektieren (patho-)physiologisch humane GI-Erkrankungen und sind immer noch unerlässlich für die Untersuchung der Pathogenese von GI-Erkrankungen.

- PDX-Modelle bewahren biologische Merkmale des ursprünglichen Tumors und eignen sich daher ideal als präklinisches Modell.

- Humane Organoide isoliert aus adultem Gewebe (z. B. Tumorpatienten) sind einfach $\mathrm{zu}$ etablieren und eignen sich für die Krankheitsmodellierung (z. B. Infektionen, Regeneration, Karzinom) und das zeitnahe Medikamentenscreening für die personalisierte Medizin und sollten daher standardisiert in der Therapieplanung angedacht werden.

- Organoide aus pluripotenten Stammzellen in Kombination mit Organs-on-a-ChipTechnologie könnten zukunftsorientiert die Modellierung von humanen gastrointestinalen Krankheiten erlauben.
- Patienten profitieren bereits heute von der Organoidtechnologie durch individualisierte Verbesserung der Therapie sowie Identifizierung neuer Biomarker für die Früherkennung von gastrointestinalen Erkrankungen.

Korrespondenzadresse

Prof. Dr. Alexander Kleger

Abteilung für Innere Medizin I, Universitätsklinikum Ulm

Albert-Einstein-Allee 23, 89081 Ulm,

Deutschland

alexander.kleger@uni-ulm.de

\section{Einhaltung ethischer Richtlinien}

Interessenkonflikt. F. Arnold und A. Kleger geben an, dass kein Interessenkonflikt besteht.

Für diesen Beitrag wurden von den Autoren keine Studien an Menschen oder Tieren durchgeführt.

The supplement containing this article is not sponsored by industry.

\section{Literatur}

1. Arnold F, Gout J, Wiese H et al (2021) RINT1 regulates SUMOylation and the DNA damage response to preserve cellular homeostasis in pancreatic cancer. Cancer Res 81:1758-1774

2. Arnold F, Mahaddalkar PU, Kraus JM et al (2021) Functional genomic screening during somatic cell reprogramming identifies DKK3 as a roadblock of organ regeneration. Adv Sci 8:2100626

3. Beutel AK, Schütte L, Scheible J et al (2021) A prospective feasibility trial to challenge patientderived pancreatic cancer organoids in predicting treatment response. Cancers (Basel) 13(11):2539

4. Breunig M, Merkle J, Wagner $M$ et al (2021) Modeling plasticity and dysplasia of pancreatic ductal organoids derived from human pluripotent stem cells. Cell Stem Cell 28:1105-1124.e19

5. Bürtin F, Mullins CS, Linnebacher M (2020) Mouse models of colorectal cancer: past, present and future perspectives. World J Gastroenterol 26:1394-1426

6. Dekkers JF, Berkers G, Kruisselbrink E et al (2016) Characterizing responses to CFTR-modulating drugs using rectal organoids derived from subjects with cystic fibrosis. Sci Transl Med 8:344ra384

7. Doyle A, McGarry MP, Lee NA et al (2012) The construction of transgenic and gene knockout/ knockin mouse models of human disease. Transgenic Res 21:327-349

8. Elyada E, Bolisetty M, Laise P et al (2019) Crossspecies single-cell analysis of pancreatic ductal adenocarcinoma reveals antigen-presenting cancer-associated fibroblasts. Cancer Discov 9:1102-1123

9. Frappart PO, WalterK, Gout J et al (2020) Pancreatic cancer-derived organoids - a disease modeling tool to predict drug response. United European Gastroenterolj 8:594-606

10. Gout J, Perkhofer L, Morawe M et al (2021) Synergistic targeting and resistance to PARP inhibition 
in DNA damage repair-deficient pancreatic cancer. Gut 70:743-760

11. Hentschel V, Arnold F, Seufferlein T et al (2021) Enteropathogenic infections: organoids go bacterial. Stem Cells Int. https://doi.org/10.1155/2021/ 8847804

12. Hentschel V, Groß R, Krüger J et al (2021) SARSCoV- 2 and the digestive tract-organoids to model gastrointestinal infection. Z Gastroenterol. https://doi.org/10.1055/a-1500-8420

13. Hingorani SR, Petricoin EF, Maitra A et al (2003) Preinvasive and invasive ductal pancreatic cancer and its early detection in the mouse. Cancer Cell 4:437-450

14. Hingorani SR, Wang L, Multani AS et al (2005) Trp53R172H and KrasG12D cooperate to promote chromosomal instability and widely metastatic pancreatic ductal adenocarcinoma in mice. Cancer Cell 7:469-483

15. Hofer M, Lutolf MP (2021) Engineering organoids. Nat Rev Mater. https://doi.org/10.1038/s41578021-00279-y

16. Hohwieler M, Illing A, Hermann PC et al (2017) Human pluripotent stem cell-derived acinar/ ductal organoids generate human pancreas upon orthotopic transplantation and allow disease modelling. Gut 66:473-486

17. Huang L, Desai R, Conrad DN et al (2021) Commitment and oncogene-induced plasticity of human stem cell-derived pancreatic acinar and ductal organoids. Cell Stem Cell 28:1090-1104.e6

18. Izumchenko E, Paz K, Ciznadija D et al (2017) Patient-derived xenografts effectively capture responses to oncology therapy in a heterogeneous cohort of patients with solid tumors. Ann Oncol 28:2595-2605

19. Kleger A, Mahaddalkar PU, Katz SF et al (2012) Increased reprogramming capacity of mouse liver progenitorcells, compared with differentiated liver cells, requires the BAF complex. Gastroenterology 142:907-917

20. Krüger J, Groß R, Conzelmann C et al (2021) Drug inhibition of SARS-CoV-2 replication in human pluripotent stem cell-derived intestinal organoids. Cell Mol Gastroenterol Hepatol 11:935-948

21. Lee AYL, Dubois CL, Sarai K et al (2019) Cell of origin affects tumour development and phenotype in pancreatic ductal adenocarcinoma. Gut 68:487-498

22. Mueller S, Engleitner T, Maresch R et al (2018) Evolutionary routes and KRAS dosage define pancreatic cancer phenotypes. Nature 554:62-68

23. Perkhofer L, Frappart PO, Müller M et al (2018) Importance of organoids for personalized medicine. Per Med 15:461-465

24. Perkhofer L, Schmitt A, Romero Carrasco MC et al (2017) ATM deficiency generating genomic instability sensitizes pancreatic ductal adenocarcinoma cells to therapy-induced DNA damage. Cancer Res 77:5576-5590

25. Russell R, llg M, Lin Q et al (2015) A dynamic role of TBX3 in the pluripotency circuitry. Stem Cell Rep 5:1155-1170

26. Russell R, Perkhofer L, Liebau S et al (2015) Loss of ATM accelerates pancreatic cancer formation and epithelial-mesenchymal transition. Nat Commun 6:7677

27. Sato T, Vries RG, Snippert HJ et al (2009) Single Lgr5 stem cells build crypt-villus structures in vitro without a mesenchymal niche. Nature 459:262-265

28. SchwankG, Koo BK, SasselliVetal (2013) Functional repair of CFTR by CRISPR/Cas9 in intestinal stem

\section{Model systems in gastroenterological research. From animal models to human organoids to the clinic}

Over the last few decades, various models have been established within gastroenterological research that have significantly contributed to a better understanding of the (patho)physiological processes of various gastrointestinal (GI) diseases (inflammation, organ injuries, carcinomas). This review will focus on such models including genetically engineered mouse models (GEMMs), xenografts, and organoid-based culture systems. GEMMs laid the foundation for successful modeling of such diseases. These have the decisive advantage that diseases can be assessed in their physiological environment and thus allow the examination of cell-cell communications of various cell types (epithelium, fibroblast, immune cells). However, the discrepancy between the genetic background of mice and humans reflected a pivotal disadvantage that could at least partially be circumvented by transplanting human cells into immunocompromised host animals. The time-consuming and labor-intensive generation of such xenograft models, however, considerably limits their usefulness for timely preclinical drug screenings. Thus, novel organoid-based human cell culture systems from adult stem cells or pluripotent stem cells are a promising human tool for modeling Gl diseases. The first results already show their usefulness in the regulation of adult tissue homeostasis, regeneration, and tumor development. In addition, this system can be easily established in clinical diagnostics and thus enables real-time ex vivo pharmacotyping to develop personalized therapy strategies, particularly for cancer patients.

\section{Keywords}

Mouse models · Xenografts · Organoids · Personalized medicine · Organ-on-a-Chip

cell organoids of cystic fibrosis patients. Cell Stem Cell 13:653-658

29. Seppälä TT, Zimmerman JW, Sereni E et al (2020) Patient-derived organoid pharmacotyping is a clinically tractable strategy for precision medicine in pancreatic cancer. Ann Surg 272:427-435

30. Spear S, Candido JB, McDermott JR et al (2019) Discrepancies in the tumor microenvironment of spontaneous and orthotopic murine models of pancreatic cancer uncover a new immunostimulatory phenotype for B cells. Front Immunol 10:542

31. Tiriac H, Belleau P, Engle DD et al (2018) Organoid profiling identifies common responders to chemotherapy in pancreatic cancer. Cancer Discov 8:1112-1129

32. Vlachogiannis $G$, Hedayat $S$, Vatsiou A et al (2018) Patient-derived organoids model treatment response of metastatic gastrointestinal cancers. Science 359:920-926

33. Walsh NC, Kenney LL, Jangalwe S et al (2017) Humanized mouse models of clinical disease. Annu Rev Pathol 12:187-215

34. Weidenhofer J, Colvin E, Bond D et al (2016) Anima models of pancreatic cancer and their application in clinical research. Gastrointest Cancer Targets Ther 6:31-39

35. Weidgang CE, Russell R, Tata PR et al (2013) TBX3 directs cell-fate decision toward mesendoderm. Stem Cell Rep 1:248-265

36. Wiedenmann S, Breunig M, Merkle J et al (2021) Single-cell-resolved differentiation of human induced pluripotent stem cells into pancreatic duct-like organoids on a microwell chip. Nat Biomed Eng 5(8):897-913 